\title{
OPTIMIZATION OF PRODUCTION COSTS WITH SIMPLEX METHOD
}

\author{
Firmansyah $^{1}$, Dedy Juliandri Panjaitan", Madyunus Salayan, \\ Alistraja Dison Silalahi \\ ${ }^{1}$ Department of Mathematics Education, FKIP, University Muslim Nusantara Alwashliyah, Indonesia \\ ${ }^{2}$ Accounting Department, Faculty of Economics, University Muslim Nusantara Alwashliyah, Indonesia \\ Corresponding Author : dedyjuliandri@umnaw.ac.id
}

\begin{abstract}
.
One of the problems in the company is my resource limitation, the time of production and the tools used in the production process. Companies in making decisions in production planning in the presence of these limitations, should seek to maximize the profit generated. However, in the production process a production planning reference is required to maximize the results obtained and minimize the production costs used. To solve the problem, needed a problem solving tool that is linear program using simplex method. Simplex method is one of the methods of linear program in solving the problem of more than two variables that can be applied into everyday life and can be used in production process planning. With one of the ultimate goal is the achievement of the optimum value with the constraints of limited resources. The results of the simplex method have decreased costs incurred less than the usual costs incurred every month and the results obtained using the simplex method can be used as a reference in making decisions to get optimal results on production costs in the company.
\end{abstract}

\section{Keywords: Optimization, Minimization, Simplex Method}

\subsection{INTRODUCTION}

The scope of mathematics is very broad, the application of mathematics in life has spread wide enough because it has positive effect with many benefits. In the production sector, which converts raw materials into new varied products expect maximum profit with minimal production costs.

Linear program is one of the solution problem in determining the optimal solution. "The problem of linear programming is basically concerned with the determination of the optimal allocation of limited resources (limited resources) to meet an objective (objective)" [1]

There are several problem solving methods in the linear program that are graphical method, algebraic method, gauss jhordan method, and simplex method.

"Most linear programming problems in the real world have more than two variables that lead to completion with less effective chart methods" [2]. The algebraic method will be more complicated in finding problem solving if more than three variables, as well as the gausss jordan method, must be more thorough in the process to obtain the minimum solution.

"In 1947 a mathematician from the United States named George D. Dantzig devised a way of deciphering and solving linear programming problems with Simplex Methods" [3]. With the simplex method will be in the final result which is the best value in minimizing profit. 
Before performing iterative calculations to determine the optimal solution, at the pre-analysis stage, determine the decision variable of the real problem. Then formulate the problem into the standard form of linear programming so that the formation of objective function and constraint function, change the inequality into the form of linear equation by adding slack variable, surplus variable and additional variable. Presents the data into the initial simplex table and determines in advance the initial feasible baseline settlement that provides the zero goal function. Specifies which variables go into the decision variables and that comes out of the base variable, based on key and key row columns. Perform calculations to generate a new split in the simplex table by iterating the simplex method until the optimal value of the destination function is reached. When we have obtained the optimal value of the objective function then we are finished in the process of simplex analysis.

\subsection{RESEARCH OBJECTIVES}

The purpose of this study is to optimize production costs as a limited resource in this case minimizing costs.

\subsection{METHODS}

This study uses a study of literature studies followed by case study research. Begin by collecting various sources concerned with linear program material and simplex methods such as journals, books, theses, and the internet. Discusses the material of simplex mathematics, slack variables, surplus variables, additional variables and materials related to this research. The next step collects data from the Business Entity owner and takes samples needed in data processing. The data taken is secondary data.

\section{LITERATURE REVIEW}

Rumahorbo [4] From the results of his research has concluded that the simplex method can be used as solution in solving linear program problem more than two variables consistently in case of maximization and minimization.

Sunarsih [5] also argues that the most successful technique in solving linear programming problems with the large number of decision and limiting variables can be used the simplex method.

Conclusion of Sukanta [6] in Simplex Method Linear Program In Polyster Material In Indonesia that the simplex method can be taken into consideration for use in minimizing costs with the use of materials to be more optimal.

In Chandra's study [5] also says that the number of iterations is not influenced by the number of variables, but depends on the value of the objective function of the previous iteration.

\section{RESULT AND DISCUSSION}

\subsection{Simplex method algorithm}

Simplex method algorithm in analyzing data as follows:

1. Identify decision variables and formulate them into mathematical symbols.

2. Identify the objective function to be achieved and the function of the boundary into the mathematical model. 
3. Function objectives and boundary functions are formulated into standard form of simplex method by adding slack variables, surplus variables, and additional variables.

4. Creating initial table of simplex method Initial table

5. Enter the value of each variable into the simplex initial table

6. Specify a key column based on the largest $\mathrm{z}$ value.

7. Determine the solution ratio

$$
=\frac{S}{\text { nthe key row column value }}
$$

8. Determining the lock row based on the smallest ratios (without $\mathrm{z}$ row)

9. Specifies the cell element that is the slice of the key column and the lock row

10. perform a stages (iteration) that begins by specifying a new row of keys

new row of keys $=\frac{\text { Key Lock lines }}{\text { elemen cell }}$

11. transform a line other than the lock line new row besides row lock = old row $-[$ (old column value) $\mathrm{x}$ (new row key)

(if the coefficient on the $\mathrm{z}$ row still exists that is positive, then back to the numbers 6 - 11)

12. Testing the optimality, until all the coefficients on the z-row is no longer a positive value, which means the table is optimal.

\subsection{Mathematical Model of Simplex Method}

Minimize objective function (purpose function)

$z-c_{1} x_{1}-c_{2} x_{2}-c_{3} x_{3}+M\left(r_{1}+r_{2}+r_{3}+r_{4}+r_{6}+r_{7}+r_{9}\right)=0$

With constraints

$a_{11} x_{1}+a_{12} x_{2}+a_{13} x_{3}-s_{1}+r_{1}=l_{1}$

$a_{21} x_{1}+a_{22} x_{2}+a_{23} x_{3}-s_{2}+r_{2}=l_{2}$

$a_{31} x_{1}+a_{32} x_{2}+a_{33} x_{3}-s_{3}+r_{3}=l_{3}$

$a_{41} x_{1}+a_{42} x_{2}+a_{43} x_{3}-s_{4}+r_{4}=l_{4}$

$a_{51} x_{1}+a_{52} x_{2}+a_{53} x_{3}+s_{5}=l_{5}$

$a_{61} x_{1}+a_{62} x_{2}+a_{63} x_{3}-s_{6}+r_{6}=l_{6}$

$a_{71} x_{1}+a_{72} x_{2}+a_{73} x_{7}-s_{7}+r_{7}=l_{7}$

$a_{81} x_{1}+a_{82} x_{2}+a_{83} x_{8}+s_{8}=l_{8}$

$a_{91} x_{1}+a_{92} x_{2}+a_{93} x_{8}-s_{9}+r_{9}=l_{9}$

Description

$\mathrm{Z}$ : minimal production costs

$x_{n}:$ the number of production

$c_{1}, c_{2}, c_{3}$ : production cost $1 \mathrm{~kg}$ of product

$a_{11}, a_{12}, a_{13}$ : Material A for $1 \mathrm{~kg}$ of product.

$a_{21}, a_{22}, a_{23}$ : Material $\mathrm{B}$ for $1 \mathrm{~kg}$ of product.

$a_{31}, a_{32}, a_{33}$ : Material $\mathrm{C}$ for $1 \mathrm{~kg}$ of product.

$a_{41}, a_{42}, a_{43}$ : Material D for $1 \mathrm{~kg}$ of product.

$a_{51}, a_{52}, a_{53}$ : Material $\mathrm{E}$ for $1 \mathrm{~kg}$ of product.

$a_{61}, a_{62}, a_{63}$ : depreciation material 1 (Fuel oil) for $1 \mathrm{~kg}$ of product 
$a_{71}, a_{72}, a_{73}$ : depreciation materia 2 ((Firewood for $1 \mathrm{~kg}$ of product.)

$a_{81}, a_{82}, a_{83}$ : wages for $1 \mathrm{~kg}$ of product.

$a_{91}, a_{92}, a_{93}$ : packaging cost (sack) for $1 \mathrm{~kg}$ of product.

$s_{5}, s_{8}:$ Slack Variable

$s_{1}, s_{2}, s_{3}, s_{4}, s_{6}, s_{7}, s_{9} \quad$ : variable surplus

$r_{1}, r_{2}, r_{3}, r_{4}, r_{6}, r_{7}, r_{9} \quad:$ additional variables

$l_{1}, l_{2}, l_{3}, l_{4}, l_{5}, l_{6}, l_{7}, l_{8}, l_{9}:$ Limitation of resources

3.2 Decision Variables

In this study, which became a decision, that is:

$x_{1}:$ the number of square opak production

$x_{2}:$ the number of animal feed production

$x_{3}$ : the number of round opak production

\subsection{Function Goals}

The objectives to be achieved can be seen in the following table.

\begin{tabular}{clc}
\hline No & Produk & $\begin{array}{c}\text { Production Cost 1 kg } \\
\text { (Rp) }\end{array}$ \\
\hline 1 & Square Opak & 4035 \\
\hline 2 & Animal Feed & 1902 \\
\hline 3 & Round Opak & 5146 \\
\hline
\end{tabular}

\subsection{Function Constraints}

With the constraints and limitations of resources owned can be seen in the table below.

Table 2. Production Constraints Table

\begin{tabular}{clccccc}
\hline No & Product & Object A & Object B & Object C & Object D & Object E \\
\hline 1 & Square Opak & 3 & 3,75 & 0,9 & 0,45 & $0 \mathrm{gr}$ \\
\hline 2 & Animal Feed & 3,333333333 & 0 & 0 & 0 & $0 \mathrm{gr}$ \\
\hline 3 & Round Opak & 3 & 6 & 2 & 0,45 & $1 \mathrm{gr}$ \\
\hline & & $91500 \mathrm{~kg}$ & $67500 \mathrm{gr}$ & $17600 \mathrm{gr}$ & $7425 \mathrm{gr}$ & $2500 \mathrm{gr}$ \\
\hline
\end{tabular}

Table 3. Table Resource Limits

\begin{tabular}{clcccc}
\hline No & Product & fuel oil $(\mathbf{m l})$ & firewood $\left(\mathbf{c m}^{\mathbf{3}}\right)$ & Pay $(\mathbf{R p})$ & packaging (Rp) \\
\hline 1 & Square Opak & 6,9231 & 0,3 & 7,05 & 9 \\
\hline 2 & Animal Feed & 16,6667 & 0 & 7,3333 & 6 \\
\hline 3 & Round Opak & 15,3846 & 1,04 & 15 & 9 \\
\hline
\end{tabular}

Subject to 


$$
\begin{array}{rl}
3 x_{1}+3,333333333 x_{2} & +3 x_{3} \leq 91500 \\
3,75 x_{1} & +6 x_{3} \leq 67500 \\
0,9 x_{1} & +2 x_{3} \leq 17600 \\
0,45 x_{1} & 0,45 x_{3} \leq 7550 \\
& x_{3} \leq 2500 \\
6,9231 x_{1}+16,6667 x_{2}+15,3846 x_{3} & \geq 345384,6 \\
0,3 x_{1}+1,04 x_{3} & \geq 6800 \\
7,05 x_{1}+7.3333 x_{2}+15 x_{3} & \leq 233850 \\
9 x_{1}+6 x_{2}+9 x_{3} & \geq 224100
\end{array}
$$

Formulation of objective functions and constraint functions by adding slack variables, surplus variables, and additional variables as follows:

With Constraint

$$
\begin{aligned}
& 3 x_{1}+3,3333 x_{2}+3 x_{3}-s_{1}+r_{1}=91500 \\
& \rightarrow r_{1}=91500-3 x_{1}-3,3333 x_{2}-3 x_{3}+s_{1} \\
& 3,75 x_{1}+6 x_{3}-s_{2}+r_{2}=67500 \\
& \rightarrow r_{2}=67500-3,75 x_{1}-6 x_{3}+s_{2} \\
& 0,9 x_{1}+2 x_{3}-s_{3}+r_{3}=17600 \\
& \rightarrow r_{3}=17600-0,9 x_{1}-2 x_{3}+s_{3} \\
& 0,45 x_{1}+0,5 x_{3}-s_{4}+r_{4}=7550 \\
& \rightarrow r_{4}=7550-0,45 x_{1}-0,5 x_{3}+s_{4} \\
& \rightarrow r_{7}=6800-0,3 x_{1}-1,04 x_{3}+s_{7} \\
& 6,9231 x_{1}+16,6667 x_{2}+15,3846 x_{3}-s_{6}+r_{6}=345384,6 \\
& x_{3}+s_{5}=2500
\end{aligned}
$$


$7,05 x_{1}+7.3333 x_{2}+15 x_{3}+s_{8}=233850$

$$
\begin{aligned}
9 x_{1} & +6 x_{2}+9 x_{3}-s_{9}+r_{9}=224100 \\
& \rightarrow r_{9}=224100-9 x_{1}-6 x_{2}-9 x_{3}+s_{9}
\end{aligned}
$$

Minimize

$$
\begin{array}{rl}
z= & 4035 x_{1}+1902 x_{2}+5146 x_{3}+M r_{1}+M r_{2}+M r_{3}+M r_{4}+M r_{6}+M r_{7}+M r_{9} \\
z= & 4035 x_{1}+1902 x_{2}+5146 x_{3}+M\left(91500-3 x_{1}-3,3333 x_{2}-3 x_{3}+s_{1}\right) \\
& +M\left(67500-3,75 x_{1}-6 x_{3}+s_{2}\right)+M\left(17600-0,9 x_{1}-2 x_{3}+s_{3}\right) \\
& +M\left(7550-0,45 x_{1}-0,5 x_{3}+s_{4}\right)+M\left(345384,6-6,9231 x_{1}-16,6667 x_{2}-15,3846 x_{3}+\right. \\
& \left.s_{6}\right)+M\left(6800-0,3 x_{1}-1,04 x_{3}+s_{7}\right)+M\left(224100-9 x_{1}-6 x_{2}-9 x_{3}+s_{9}\right) \\
Z= & 4035 x_{1}+1902 x_{2}+5146 x_{3}+91500 M-3 M x_{1}-3,3333 M x_{2} \\
& -3 M x_{3}+M s_{1}+67500 M-3,75 M x_{1}-6 M x_{3}+M s_{2}+17600 M \\
& -0,9 M x_{1}-2 M x_{3}+M s_{3}+7550 M-0,45 M x_{1}-0,5 M x_{3}+M s_{4} \\
& +345384,6 M-6,9231 M x_{1}-16,6667 M x_{2}-15,3846 M x_{3}+M s_{6} \\
& +6800 M-0,3 M x_{1}-1,04 M x_{3}+M s_{7}+224100 M-9 M x_{1}-6 M x_{2} \\
& -9 M x_{3}+M s_{9} \\
z= & 4035 x_{1}-3 M x_{1}-3,75 M x_{1}-0,9 M x_{1}-0,45 M x_{1}-6,9231 M x_{1} \\
& \left.-0,3 M x_{1}-9 M x_{1}\right)+\left(1902 x_{2}-3,3333 M x_{2}-16,6667 M x_{2}-6 M x_{2}\right) \\
& +\left(5146 x_{3}-3 M x_{3}-6 M x_{3}-2 M x_{3}-0,5 M x_{3}-15,3846 M x_{3}\right. \\
& -1,04 M x_{3}-9 M x_{3}+91500 M+67500 M+17600 M+7550 M \\
& +345384,6 M+6800 M+224100 M+M s_{1}+M s_{2}+M s_{3}+M s_{4}+M s_{6} \\
& +M s_{7}+M s_{9} \\
z= & 4035-24,3231 M) x_{1}+(1902-26 M) x_{2}+(5146-36,9246 M) x_{3} \\
& +760434,6 M+M s_{1}+M s_{2}+M s_{3}+M s_{4}+M s_{6}+M s_{7}+M s_{9} \\
Z- & (4035-24,3231 M) x_{1}-(1902-26 M) x_{2}-(5146-36,9246 M) x_{3} \\
-M s_{1} & -M s_{2}-M s_{3}-M s_{4}-M s_{6}-M s_{7}-M s_{9}=760434,6 M \\
C & 0 M P L E T(N)
\end{array}
$$

\begin{tabular}{|c|c|c|c|c|c|c|c|c|c|c|c|c|c|c|c|c|c|c|c|c|c|c|}
\hline VD & $z$ & $\mathrm{X} 1$ & $\mathrm{X2}$ & X3 & $r 1$ & s1 & r2 & s2 & r3 & s3 & r4 & s4 & s5 & r6 & s6 & $r 7$ & s7 & s8 & r9 & s9 & $S$ & \\
\hline$z$ & 1 & $4035+24.3231 \mathrm{M}$ & $\begin{array}{c}- \\
1902+26 \mathrm{M}\end{array}$ & $\begin{array}{c}- \\
5146+36.9246 \mathrm{M}\end{array}$ & 0 & $\bar{M}$ & 0 & $\bar{M}$ & 0 & $\bar{M}$ & 0 & $\bar{M}$ & 0 & 0 & $\bar{M}$ & 0 & $\bar{M}$ & 0 & 0 & $\bar{M}$ & $760434.6 \mathrm{M}$ & is \\
\hline$r 1$ & 0 & 3 & 3.3333 & 3 & 1 & -1 & 0 & 0 & 0 & 0 & 0 & 0 & 0 & 0 & 0 & 0 & 0 & 0 & 0 & 0 & 91500 & 30500 \\
\hline r2 & 0 & 3.75 & 0 & 6 & 0 & 0 & 1 & -1 & 0 & 0 & 0 & 0 & 0 & 0 & 0 & 0 & 0 & 0 & 0 & 0 & 67500 & 11250 \\
\hline r3 & 0 & 0.9 & 0 & 2 & 0 & 0 & 0 & 0 & 1 & -1 & 0 & 0 & 0 & 0 & 0 & 0 & 0 & 0 & 0 & 0 & 17600 & 8800 \\
\hline$r 4$ & 0 & 0.45 & 0 & 0.5 & 0 & 0 & 0 & 0 & 0 & 0 & 1 & -1 & 0 & 0 & 0 & 0 & 0 & 0 & 0 & 0 & 7550 & 15100 \\
\hline
\end{tabular}

4 COMPLETION WITH SIMPLEX TABLE

The function objectives and function constraints have been formulated into the standard

form of the simplex method by adding the slack variable, arranged into the simplex initial table.

Table 4. initial table minimization 


\begin{tabular}{|c|c|c|c|c|c|c|c|c|c|c|c|c|c|c|c|c|c|c|c|c|c|c|}
\hline s5 & 0 & 0 & 0 & 1 & 0 & 0 & 0 & 0 & 0 & 0 & 0 & 0 & 1 & 0 & 0 & 0 & 0 & 0 & 0 & 0 & 2500 & 2500 \\
\hline r6 & 0 & 6.9231 & 16.6667 & 15.3846 & 0 & 0 & 0 & 0 & 0 & 0 & 0 & 0 & 0 & 1 & -1 & 0 & 0 & 0 & 0 & 0 & 345384.6 & 22450.0215 \\
\hline r7 & 0 & 0.3 & 0 & 1.04 & 0 & 0 & 0 & 0 & 0 & 0 & 0 & 0 & 0 & 0 & 0 & 1 & -1 & 0 & 0 & 0 & 6800 & 6538.46154 \\
\hline s8 & 0 & 7.05 & 7.3333 & 15 & 0 & 0 & 0 & 0 & 0 & 0 & 0 & 0 & 0 & 0 & 0 & 0 & 0 & 1 & 0 & 0 & 233850 & 15590 \\
\hline r9 & 0 & 9 & 6 & 9 & 0 & 0 & 0 & 0 & 0 & 0 & 0 & 0 & 0 & 0 & 0 & 0 & 0 & 0 & 1 & -1 & 224100 & 24900 \\
\hline
\end{tabular}

\section{Table 5. first iteration}

\begin{tabular}{|c|c|c|c|c|c|c|c|c|c|c|c|c|c|c|c|c|c|c|c|c|c|c|}
\hline VD & $z$ & $\mathrm{x} 1$ & X2 & X3 & r1 & s1 & r2 & s2 & r3 & s3 & r4 & s4 & s5 & r6 & s6 & r7 & s7 & s8 & r9 & s9 & $\mathrm{s}$ & \\
\hline $\mathrm{z}$ & 1 & $\begin{array}{c}-4035+24 . \\
323076923 \mathrm{M}\end{array}$ & $-1902+26 \mathrm{M}$ & 0 & 0 & $\overline{\mathrm{M}}$ & 0 & $\bar{M}$ & 0 & $\bar{M}$ & 0 & M & $\begin{array}{c}5146- \\
36.9246 \mathrm{M}\end{array}$ & 0 & $\overline{\mathrm{M}}$ & 0 & $\bar{M}$ & 0 & 0 & $\overline{\mathrm{M}}$ & $12865000+668123.1 \mathrm{M}$ & Rs \\
\hline r1 & 0 & 3 & 3.333333333 & 0 & 1 & -1 & 0 & 0 & 0 & 0 & 0 & 0 & -3 & 0 & 0 & 0 & 0 & 0 & 0 & 0 & 84000 & 25200 \\
\hline r2 & 0 & 3.75 & 0 & 0 & 0 & 0 & 1 & -1 & 0 & 0 & 0 & 0 & -6 & 0 & 0 & 0 & 0 & 0 & 0 & 0 & 52500 & \\
\hline r3 & 0 & 0.9 & 0 & 0 & 0 & 0 & 0 & 0 & 1 & -1 & 0 & 0 & -2 & 0 & 0 & 0 & 0 & 0 & 0 & 0 & 12600 & \\
\hline$r 4$ & 0 & 0.45 & 0 & 0 & 0 & 0 & 0 & 0 & 0 & 0 & 1 & -1 & -0.5 & 0 & 0 & 0 & 0 & 0 & 0 & 0 & 6300 & \\
\hline$x 3$ & 0 & 0 & 0 & 1 & 0 & 0 & 0 & 0 & 0 & 0 & 0 & 0 & 1 & 0 & 0 & 0 & 0 & 0 & 0 & 0 & 2500 & \\
\hline r6 & 0 & 6.9231 & 16.6667 & 0 & 0 & 0 & 0 & 0 & 0 & 0 & 0 & 0 & -15.3846 & 1 & -1 & 0 & 0 & 0 & 0 & 0 & 306923.1 & 18415.3492 \\
\hline$r 7$ & 0 & 0.3 & 0 & 0 & 0 & 0 & 0 & 0 & 0 & 0 & 0 & 0 & -1.04 & 0 & 0 & 1 & -1 & 0 & 0 & 0 & 4200 & \\
\hline s8 & 0 & 7.05 & 7.3333 & 0 & 0 & 0 & 0 & 0 & 0 & 0 & 0 & 0 & -15 & 0 & 0 & 0 & 0 & 1 & 0 & 0 & 196350 & 26775.1217 \\
\hline r9 & 0 & 9 & 6 & 0 & 0 & 0 & 0 & 0 & 0 & 0 & 0 & 0 & -9 & 0 & 0 & 0 & 0 & 0 & 1 & -1 & 201600 & 33600 \\
\hline
\end{tabular}

Table 6. Second Iteration

\begin{tabular}{|c|c|c|c|c|c|c|c|c|c|c|c|c|c|c|c|c|c|c|c|c|c|c|}
\hline VD & $z$ & $\mathrm{x} 1$ & X2 & $\mathrm{x} 3$ & $r 1$ & s1 & r2 & s2 & r3 & s3 & $\mathrm{r4}$ & s4 & s5 & r6 & s6 & $\mathrm{r} 7$ & s7 & s8 & r9 & s9 & $s$ & \\
\hline z & 1 & $\begin{array}{l}-3244.937408+ \\
13.52306252 \mathrm{M}\end{array}$ & 0 & 0 & 0 & $-\mathrm{M}$ & 0 & $-\mathrm{M}$ & 0 & $-M$ & 0 & $-M$ & $\begin{array}{c}3390.312959- \\
12.924672 \mathrm{M}\end{array}$ & $\begin{array}{l}114.1197718- \\
1.55999688 \mathrm{M}\end{array}$ & $\begin{array}{l}-114.1197718 \\
+0.55999688 \mathrm{M}\end{array}$ & 0 & $-M$ & 0 & 0 & $-M$ & $\begin{array}{l}47890994.12+ \\
189324.0216 \mathrm{M}\end{array}$ & Rs \\
\hline $\mathrm{r} 1$ & 0 & 1.615382769 & 0 & 0 & 1 & -1 & 0 & 0 & 0 & 0 & 0 & 0 & 0.076913846 & -0.1999996 & 0.1999996 & 0 & 0 & 0 & 0 & 0 & 22615.50278 & 14000.0891 \\
\hline r2 & 0 & 3.75 & 0 & 0 & 0 & 0 & 1 & -1 & 0 & 0 & 0 & 0 & -6 & 0 & 0 & 0 & 0 & 0 & 0 & 0 & 52500 & 14000 \\
\hline $\mathrm{r3}$ & 0 & 0.9 & 0 & 0 & 0 & 0 & 0 & 0 & 1 & -1 & 0 & 0 & -2 & 0 & 0 & 0 & 0 & 0 & 0 & 0 & 12600 & 14000 \\
\hline r4 & 0 & 0.45 & 0 & 0 & 0 & 0 & 0 & 0 & 0 & 0 & 1 & -1 & -0.5 & 0 & 0 & 0 & 0 & 0 & 0 & 0 & 6300 & 14000 \\
\hline x3 & 0 & 0 & 0 & 1 & 0 & 0 & 0 & 0 & 0 & 0 & 0 & 0 & 1 & 0 & 0 & 0 & 0 & 0 & 0 & 0 & 2500 & \\
\hline $\mathrm{x} 2$ & 0 & 0.415385169 & 1 & 0 & 0 & 0 & 0 & 0 & 0 & 0 & 0 & 0 & -0.923074154 & 0.05999988 & -0.05999988 & 0 & 0 & 0 & 0 & 0 & 18415.34917 & 44333.1889 \\
\hline $\mathrm{r} 7$ & 0 & 0.3 & 0 & 0 & 0 & 0 & 0 & 0 & 0 & 0 & 0 & 0 & -1.04 & 0 & 0 & 1 & -1 & 0 & 0 & 0 & 4200 & 14000 \\
\hline s8 & 0 & 4.003855938 & 0 & 0 & 0 & 0 & 0 & 0 & 0 & 0 & 0 & 0 & -8.230820308 & -0.43999712 & 0.43999712 & 0 & 0 & 1 & 0 & 0 & 61304.71994 & 15311.42 \\
\hline r9 & 0 & 6.507688985 & 0 & 0 & 0 & 0 & 0 & 0 & 0 & 0 & 0 & 0 & -3.461555077 & -0.35999928 & 0.35999928 & 0 & 0 & 0 & 1 & -1 & 91107.90498 & 14000.0398 \\
\hline
\end{tabular}

Table 7. Third iteration

\begin{tabular}{|c|c|c|c|c|c|c|c|c|c|c|c|c|c|c|c|c|c|c|c|c|c|c|}
\hline VD & $z$ & $\mathrm{X} 1$ & $\mathrm{x} 2$ & X3 & $\mathrm{r} 1$ & s1 & $\mathrm{r2}$ & s2 & r3 & s3 & r4 & s4 & s5 & r6 & s6 & $r 7$ & s7 & s8 & r9 & s9 & $S$ & \\
\hline $\mathrm{z}$ & 1 & 0 & 0 & 0 & 0 & 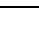 & 865.3166422 & -865.3166422 & 0 & 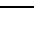 & 0 & 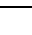 & $-1801.586894+$ & 114.1197718 & -114.1197718 & 0 & 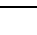 & 0 & 0 & . & 93320117.83 & \\
\hline
\end{tabular}




\begin{tabular}{|c|c|c|c|c|c|c|c|c|c|c|c|c|c|c|c|c|c|c|c|c|c|c|}
\hline & & & & & & & & & & & & & \multicolumn{10}{|c|}{$\begin{array}{r}\text { Volume } 4 \text { Number } 2 \\
-1849 \text { | e-ISSN: } 2549-3434\end{array}$} \\
\hline & & & & & & M & $\begin{array}{c}- \\
3.606150006 \mathrm{M}\end{array}$ & $+2.606150006 \mathrm{M}$ & & M & & M & $8.712228037 \mathrm{M}$ & $\begin{array}{c}- \\
1.55999688 \mathrm{M}\end{array}$ & $+0.55999688 \mathrm{M}$ & & M & & & M & $+1.146275754 \mathrm{M}$ & \\
\hline$r 1$ & 0 & 0 & 0 & 0 & 1 & -1 & $-0,430768738$ & 0,430768738 & 0 & 0 & 0 & 0 & 2,66153 & $-0,1999996$ & 0,1999996 & 0 & 0 & 0 & 0 & 0 & 0,144003912 & 0,054105764 \\
\hline $\mathrm{x} 1$ & 0 & 1 & 0 & 0 & 0 & 0 & 0,266666667 & $-0,266666667$ & 0 & 0 & 0 & 0 & $-1,6$ & 0 & 0 & 0 & 0 & 0 & 0 & 0 & 14000 & -8750 \\
\hline r3 & 0 & 0 & 0 & 0 & 0 & 0 & $-0,24$ & 0,24 & 1 & -1 & 0 & 0 & $-0,56$ & 0 & 0 & 0 & 0 & 0 & 0 & 0 & 0 & 0 \\
\hline$r 4$ & 0 & 0 & 0 & 0 & 0 & 0 & $-0,12$ & 0,12 & 0 & 0 & 1 & -1 & 0,22 & 0 & 0 & 0 & 0 & 0 & 0 & 0 & 0 & 0 \\
\hline$\times 3$ & 0 & 0 & 0 & 1 & 0 & 0 & 0 & 0 & 0 & 0 & 0 & 0 & 1 & 0 & 0 & 0 & 0 & 0 & 0 & 0 & 2500 & 2500 \\
\hline$x 2$ & 0 & 0 & 1 & 0 & 0 & 0 & $-0,110769378$ & 0,110769378 & 0 & 0 & 0 & 0 & $-0,2585$ & 0,05999988 & $-0,05999988$ & 0 & 0 & 0 & 0 & 0 & 12599,9568 & 48750,52233 \\
\hline $\mathrm{r} 7$ & 0 & 0 & 0 & 0 & 0 & 0 & $-0,08$ & 0,08 & 0 & 0 & 0 & 0 & $-0,56$ & 0 & 0 & 1 & -1 & 0 & 0 & 0 & 0 & 0 \\
\hline s8 & 0 & 0 & 0 & 0 & 0 & 0 & $-1,067694917$ & 1,067694917 & 0 & 0 & 0 & 0 & $-1,8247$ & $-0,43999712$ & 0,43999712 & 0 & 0 & 1 & 0 & 0 & 5250,736798 & 2877,666664 \\
\hline r9 & 0 & 0 & 0 & 0 & 0 & 0 & $-1,735383729$ & 1,735383729 & 0 & 0 & 0 & 0 & 6,95075 & $-0,35999928$ & 0,35999928 & 0 & 0 & 0 & 1 & -1 & 0,259199482 & 0,03729088 \\
\hline
\end{tabular}

Table 8. the fourth iteration

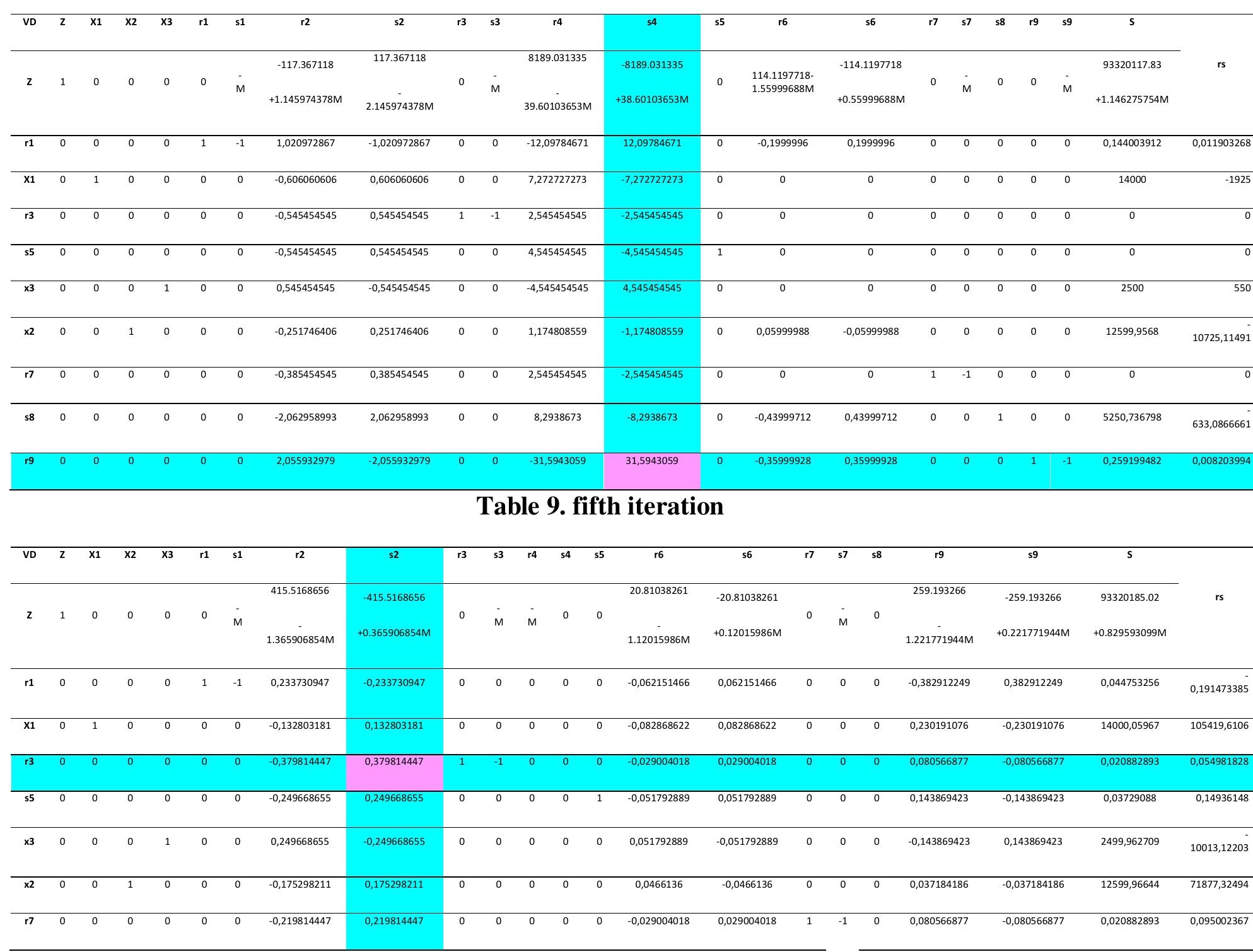




\begin{tabular}{lllllllllllll}
0 & 0 & 0 & 0 & $-0,534501056$ & 0,534501056 & 0 & 0 & 1 & 0,262511458 & $-0,262511458$ & 5250,804841 & 3447,099555 \\
\hline 0 & -1 & 1 & 0 & $-0,011394435$ & 0,011394435 & 0 & 0 & 0 & 0,031651273 & $-0,031651273$ & 0,008203994 & 0,126073896
\end{tabular}

\section{Table 10. iteration sixth}

\begin{tabular}{|c|c|c|c|c|c|c|c|c|c|c|c|c|c|c|c|c|c|c|c|c|c|c|}
\hline VD & $z$ & $\mathrm{x}_{1}$ & $x^{2}$ & x3 & $r 1$ & s1 & r2 & s2 & r3 & s3 & $r 4$ & s4 & s5 & r6 & s6 & r7 & s7 & 58 & r9 & s9 & $\mathrm{s}$ & \\
\hline$z$ & 1 & 0 & 0 & 0 & 0 & $\overline{\mathrm{M}}$ & $\bar{M}$ & 0 & $\begin{array}{c}1093.999633 \\
- \\
0.963383192 \mathrm{M}\end{array}$ & $\begin{array}{c}-1093.999633 \\
- \\
0.036616808 \mathrm{M}\end{array}$ & M & 0 & 0 & $\begin{array}{c}-10.92000198 \\
- \\
1.092217877 \mathrm{M}\end{array}$ & $\begin{array}{r}10.92000198 \\
+0.092217877 \mathrm{M}\end{array}$ & 0 & $\overline{\mathrm{M}}$ & 0 & $\begin{array}{c}347.3333995 \\
- \\
1.299388718 \mathrm{M}\end{array}$ & $\begin{array}{c}-347.3333995 \\
+0.299388718 \mathrm{M}\end{array}$ & $\begin{array}{c}93320207.86 \\
+0.809474871 \mathrm{M}\end{array}$ & rs \\
\hline $\mathrm{r} 1$ & 0 & 0 & 0 & 0 & 1 & -1 & 0 & 0 & $-0,61538193$ & $-0,61538193$ & 0 & 0 & 0 & $-0,080000015$ & 0,080000015 & 0 & 0 & 0 & $-0,333332848$ & 0,333332848 & 0,05760421 & 0,17281 \\
\hline $\mathrm{x} 1$ & 0 & 1 & 0 & 0 & 0 & 0 & 0 & 0 & $-0,349652791$ & 0,349652791 & 0 & 0 & 0 & $-0,072727286$ & 0,072727286 & 0 & 0 & 0 & 0,202020643 & $-0,202020643$ & 14000,05236 & $-69300,1$ \\
\hline s2 & 0 & 0 & 0 & 0 & 0 & 0 & -1 & 1 & 2,63286457 & $-2,63286457$ & 0 & 0 & 0 & $-0,07636365$ & 0,07636365 & 0 & 0 & 0 & 0,212121675 & $-0,212121675$ & 0,054981828 & $-0,259199$ \\
\hline s5 & 0 & 0 & 0 & 0 & 0 & 0 & 0 & 0 & $-0,657343756$ & 0,657343756 & 0 & 0 & 1 & $-0,032727279$ & 0,032727279 & 0 & 0 & 0 & 0,090909289 & $-0,090909289$ & 0,023563641 & $-0,259199$ \\
\hline$x^{3}$ & 0 & 0 & 0 & 1 & 0 & 0 & 0 & 0 & 0,657343756 & $-0,657343756$ & 0 & 0 & 0 & 0,032727279 & $-0,032727279$ & 0 & 0 & 0 & $-0,090909289$ & 0,090909289 & 2499,976436 & 27499,680 \\
\hline$\times 2$ & 0 & 0 & 1 & 0 & 0 & 0 & 0 & 0 & $-0,461536448$ & 0,461536448 & 0 & 0 & 0 & 0,060000011 & $-0,060000011$ & 0 & 0 & 0 & $-3,63636 \mathrm{E}-07$ & $3,63636 \mathrm{E}-07$ & 12599,9568 & $3,465 \mathrm{E}+1$ \\
\hline r7 & 0 & 0 & 0 & 0 & 0 & 0 & 0 & 0 & $-0,578741669$ & 0,578741669 & 0 & 0 & 0 & $-0,012218184$ & 0,012218184 & 1 & -1 & 0 & 0,033939468 & $-0,033939468$ & 0,008797093 & $-0,259199$ \\
\hline 58 & 0 & 0 & 0 & 0 & 0 & 0 & 0 & 0 & $-4,010518932$ & 4,010518932 & 0 & 0 & 0 & $-0,418179894$ & 0,418179894 & 0 & 0 & 1 & $-0,060603526$ & 0,060603526 & 5250,72109 & 86640,52 \\
\hline 54 & 0 & 0 & 0 & 0 & 0 & 0 & 0 & 0 & 0,171328122 & $-0,171328122$ & -1 & 1 & 0 & $-0,016363639$ & 0,016363639 & 0 & 0 & 0 & 0,045454645 & $-0,045454645$ & 0,01178182 & $-0,259199$ \\
\hline
\end{tabular}

Table 11. the seventh iteration

\begin{tabular}{|c|c|c|c|c|c|c|c|c|c|c|c|c|c|c|c|c|c|c|c|c|c|c|}
\hline VD & $z$ & $\mathbf{x 1}$ & $\mathrm{x} 2$ & $\mathrm{X3}$ & r1 & s1 & $r 2$ & s2 & r3 & s3 & $\mathrm{r} 4$ & s4 & s5 & r6 & s6 & $r 7$ & s7 & s8 & r9 & s9 & $s$ & \\
\hline$z$ & 1 & 0 & 0 & 0 & $\begin{array}{c}1042.001714- \\
0.898167462 \mathrm{M}\end{array}$ & $\begin{array}{l}-1042.001714- \\
0.101832538 \mathrm{M}\end{array}$ & $\bar{M}$ & 0 & $\begin{array}{l}452.7706076- \\
0.410667166 \mathrm{M}\end{array}$ & $\begin{array}{r}-1735.228659 \\
+0.516099218 \mathrm{M}\end{array}$ & $-M$ & 0 & 0 & $\begin{array}{c}-94.28015428- \\
1.020364467 \mathrm{M}\end{array}$ & $\begin{array}{r}94.28015428 \\
+0.020364467 \mathrm{M}\end{array}$ & 0 & $-M$ & 0 & M & 0 & $\begin{array}{r}93320267.89 \\
+0.757736644 \mathrm{M}\end{array}$ & rs \\
\hline s9 & 0 & 0 & 0 & 0 & 3,000004364 & $-3,000004364$ & 0 & 0 & $-1,846148475$ & $-1,846148475$ & 0 & 0 & 0 & $-0,240000393$ & 0,240000393 & 0 & 0 & 0 & -1 & 1 & 0,172812883 & $-0,09360$ \\
\hline$\overline{\mathbf{x} 1}$ & 0 & 1 & 0 & 0 & 0,60606281 & $-0,60606281$ & 0 & 0 & $-0,722612892$ & $-0,023307311$ & 0 & 0 & 0 & $-0,12121232$ & 0,12121232 & 0 & 0 & 0 & 0 & 0 & 14000,08728 & -600673 \\
\hline s2 & 0 & 0 & 0 & 0 & 0,63636595 & $-0,63636595$ & -1 & 1 & 2,241256463 & $-3,024472676$ & 0 & 0 & 0 & $-0,127272936$ & 0,127272936 & 0 & 0 & 0 & 0 & 0 & 0,091639186 & $-0,03029$ \\
\hline s5 & 0 & 0 & 0 & 0 & 0,272728264 & $-0,272728264$ & 0 & 0 & $-0,825175802$ & 0,48951171 & 0 & 0 & 1 & $-0,054545544$ & 0,054545544 & 0 & 0 & 0 & 0 & 0 & 0,039273937 & 0,080230 \\
\hline$x 3$ & 0 & 0 & 0 & 1 & $-0,272728264$ & 0,272728264 & 0 & 0 & 0,825175802 & $-0,48951171$ & 0 & 0 & 0 & 0,054545544 & $-0,054545544$ & 0 & 0 & 0 & 0 & 0 & 2499,960726 & $-5107,04$ \\
\hline$\times 2$ & 0 & 0 & 1 & 0 & $-1,09091 \mathrm{E}-06$ & $1,09091 \mathrm{E}-06$ & 0 & 0 & $-0,461535776$ & 0,461537119 & 0 & 0 & 0 & 0,060000098 & $-0,060000098$ & 0 & 0 & 0 & 0 & 0 & 12599,9568 & 27299,98 \\
\hline$r 7$ & 0 & 0 & 0 & 0 & 0,101818552 & $-0,101818552$ & 0 & 0 & $-0,641398966$ & 0,516084372 & 0 & 0 & 0 & $-0,02036367$ & 0,02036367 & 1 & -1 & 0 & 0 & 0 & 0,01466227 & 0,028410 \\
\hline 58 & 0 & 0 & 0 & 0 & $-0,181810843$ & 0,181810843 & 0 & 0 & $-3,898635824$ & 4,122402039 & 0 & 0 & 0 & $-0,403635024$ & 0,403635024 & 0 & 0 & 1 & 0 & 0 & 5250,710616 & 1273,701 \\
\hline s4 & 0 & 0 & 0 & 0 & 0,136364132 & $-0,136364132$ & 0 & 0 & 0,087412099 & $-0,255244145$ & -1 & 1 & 0 & $-0,027272772$ & 0,027272772 & 0 & 0 & 0 & 0 & 0 & 0,019636968 & $-0,07693$ \\
\hline
\end{tabular}

\section{Table 12. iteration eighth}

\begin{tabular}{|c|c|c|c|c|c|c|c|c|c|c|c|c|c|c|c|c|c|c|c|c|c|c|}
\hline VD & $z$ & $\mathrm{x} 1$ & $\mathrm{x} 2$ & $x_{3}$ & $\mathrm{r1}$ & s1 & $\mathrm{r} 2$ & s2 & r3 & s3 & $r 4$ & s4 & 55 & r6 & 56 & r7 & s7 & 58 & r9 & 59 & $\mathrm{~s}$ & \\
\hline $\mathrm{z}$ & 1 & 0 & 0 & 0 & $\begin{array}{c}1384.345872- \\
0.999988943 \mathrm{M}\end{array}$ & $\begin{array}{c}- \\
1384.345872- \\
1.1057 \mathrm{E}-05 \mathrm{M}\end{array}$ & M & 0 & $\begin{array}{r}-1703.802869 \\
+0.230750251 \mathrm{M}\end{array}$ & 0 & $\bar{M}$ & 0 & 0 & $\begin{array}{c}-162.7488489 \\
- \\
1000000211 \mathrm{M}\end{array}$ & $\begin{array}{r}162.7488489 \\
+2.114 \mathrm{E}-07 \mathrm{M}\end{array}$ & $\begin{array}{c}3362.296466- \\
1.000028768 \mathrm{M}\end{array}$ & $\begin{array}{c}3362.296466 \\
+2.87677 \mathrm{E}- \\
05 \mathrm{M}\end{array}$ & 0 & $\bar{M}$ & 0 & $\begin{array}{r}93320317.18 \\
+0.743073952 \mathrm{M}\end{array}$ & rs \\
\hline s9 & 0 & 0 & 0 & 0 & 3,364231949 & $-3,364231949$ & 0 & 0 & $-4,140575099$ & 0 & 0 & 0 & 0 & $-0,312845764$ & 0,312845764 & 3,577222206 & $\begin{array}{l}- \\
3,577222206\end{array}$ & 0 & -1 & 1 & 0,22526308 & $-0,0629715$ \\
\hline $\mathrm{x} 1$ & 0 & 1 & 0 & 0 & 0,610661121 & $-0,610661121$ & 0 & 0 & $-0,751579639$ & 0 & 0 & 0 & 0 & $-0,12213198$ & 0,12213198 & 0,045161822 & 0,045161822 & 0 & 0 & 0 & 14000,08794 & $-309998,29$ \\
\hline s2 & 0 & 0 & 0 & 0 & 1,233065726 & $-1,233065726$ & -1 & 1 & $-1,517612732$ & 0 & 0 & 0 & 0 & $-0,246612652$ & 0,246612652 & 5,860422911 & 5,860422911 & 0 & 0 & 0 & 0,177566288 & $-0,0302992$ \\
\hline s5 & 0 & 0 & 0 & 0 & 0,176152247 & $-0,176152247$ & 0 & 0 & $-0,216801819$ & 0 & 0 & 0 & 1 & $-0,035230379$ & 0,035230379 & $-0,948511013$ & 0,948511013 & 0 & 0 & 0 & 0,025366613 & 0,02674361 \\
\hline
\end{tabular}




\begin{tabular}{|c|c|c|c|c|c|c|c|c|c|c|c|c|c|c|c|c|c|c|c|c|c|c|}
\hline x3 & 0 & 0 & 0 & 1 & $-0,176152247$ & 0,176152247 & 0 & 0 & 0,216801819 & 0 & 0 & 0 & 0 & 0,035230379 & $-0,035230379$ & 0,948511013 & 0,948511013 & 0 & 0 & 0 & 2499,974633 & $-2635,683$ \\
\hline $\mathrm{x} 2$ & 0 & 0 & 1 & 0 & $-0,091057987$ & 0,091057987 & 0 & 0 & 0,11207088 & 0 & 0 & 0 & 0 & 0,078211441 & $-0,078211441$ & $-0,894305552$ & 0,894305552 & 0 & 0 & 0 & 12599,94369 & 14089,081 \\
\hline s3 & 0 & 0 & 0 & 0 & 0,197290516 & $-0,197290516$ & 0 & 0 & $-1,242818037$ & 1 & 0 & 0 & 0 & $-0,039458024$ & 0,039458024 & 1,937667666 & 1,937667666 & 0 & 0 & 0 & 0,028410606 & $-0,014662$ \\
\hline 58 & 0 & 0 & 0 & 0 & $-0,995121669$ & 0,995121669 & 0 & 0 & 1,224759786 & 0 & 0 & 0 & 0 & $-0,240973184$ & 0,240973184 & $-7,987845137$ & 7,987845137 & 1 & 0 & 0 & 5250,593497 & 657,32289 \\
\hline s4 & 0 & 0 & 0 & 0 & 0,186721381 & $-0,186721381$ & 0 & 0 & $-0,229809928$ & 0 & -1 & 1 & 0 & $-0,037344202$ & 0,037344202 & 0,494578326 & 0.494578326 & 0 & 0 & 0 & 0,026888609 & $-0,054366$ \\
\hline
\end{tabular}

Table 13. the ninth iteration

\begin{tabular}{|c|c|c|c|c|c|c|c|c|c|c|c|c|c|c|c|c|c|c|c|c|c|}
\hline VD & $z$ & $\mathrm{x} 1$ & $\mathrm{x} 2$ & $\mathrm{X3}$ & $\mathrm{r1}$ & s1 & r2 & s2 & r3 & s3 & $\mathrm{r} 4$ & s4 & 55 & r6 & 56 & r7 & s7 & s8 & r9 & s9 & $\mathrm{s}$ \\
\hline $\mathrm{z}$ & 1 & 0 & 0 & 0 & $\begin{array}{c}2008.773072- \\
0.999994286 \mathrm{M}\end{array}$ & $\begin{array}{c}2008.773072- \\
571441 \mathrm{E}- \\
06 \mathrm{M}\end{array}$ & $\dot{M}$ & 0 & $\begin{array}{c}-2472.3253 \\
+0.230756827 \mathrm{M}\end{array}$ & 0 & $M$ & 0 & $\begin{array}{c}3544.815422- \\
3.03293 \mathrm{E}- \\
05 \mathrm{M}\end{array}$ & $\begin{array}{l}-287.6340391- \\
0.999999143 \mathrm{M}\end{array}$ & $\begin{array}{c}287.6340391- \\
8.57113 \mathrm{E}- \\
07 \mathrm{M}\end{array}$ & $\bar{M}$ & 0 & 0 & $\bar{M}$ & 0 & 93320407,1 \\
\hline 59 & 0 & 0 & 0 & 0 & 4,028573975 & $-4,028573975$ & 0 & 0 & $-4,958223255$ & 0 & 0 & 0 & 3,771408195 & $-0,445713904$ & 0,445713904 & 0 & 0 & 0 & -1 & 1 & 0,320930931 \\
\hline $\mathrm{x} 1$ & 0 & 1 & 0 & 0 & 0,619048326 & $-0,619048326$ & 0 & 0 & $-0,761902308$ & 0 & 0 & 0 & 0,047613388 & $-0,123809418$ & 0,123809418 & 0 & 0 & 0 & 0 & 0 & 14000,08915 \\
\hline s2 & 0 & 0 & 0 & 0 & 2,321431224 & $-2,321431224$ & -1 & 1 & $-2,857133654$ & 0 & 0 & 0 & 6,178550203 & $-0,464285316$ & 0,464285316 & 0 & 0 & 0 & 0 & 0 & 0,334295178 \\
\hline s7 & 0 & 0 & 0 & 0 & 0,185714498 & $-0,185714498$ & 0 & 0 & $-0,228570692$ & 0 & 0 & 0 & 1,054284016 & $-0,037142825$ & 0,037142825 & -1 & 1 & 0 & 0 & 0 & 0,026743614 \\
\hline$x^{3}$ & 0 & 0 & 0 & 1 & 0 & 0 & 0 & 0 & 0 & 0 & 0 & 0 & 1 & 0 & 0 & 0 & 0 & 0 & 0 & 0 & 2500 \\
\hline $\mathbf{x 2}$ & 0 & 0 & 1 & 0 & $-0,257143494$ & 0,257143494 & 0 & 0 & 0,316482919 & 0 & 0 & 0 & $-0,942852049$ & 0,111428476 & $-0,111428476$ & 0 & 0 & 0 & 0 & 0 & 12599,91977 \\
\hline s3 & 0 & 0 & 0 & 0 & 0,557143494 & $-0,557143494$ & 0 & 0 & $-1,685712077$ & 1 & 0 & 0 & 2,042852049 & $-0,111428476$ & 0,111428476 & 0 & 0 & 0 & 0 & 0 & 0,080230843 \\
\hline 58 & 0 & 0 & 0 & 0 & $-2,478580318$ & 2,478580318 & 0 & 0 & 3,050547079 & 0 & 0 & 0 & $-8,421457452$ & 0,055717952 & $-0,055717952$ & 0 & 0 & 1 & 0 & 0 & 5250,379873 \\
\hline s4 & 0 & 0 & 0 & 0 & 0,278571747 & $-0,278571747$ & 0 & 0 & $-0,342856038$ & 0 & -1 & 1 & 0,521426024 & $-0,055714238$ & 0,055714238 & 0 & 0 & 0 & 0 & 0 & 0,040115421 \\
\hline
\end{tabular}

From the calculation result of simplex method gives minimum value of $\mathrm{z}=93320407,1$ when $X \_1=$ $1400,08915 \approx X \_1=1400, X \_2=12599,91977 \approx X \_2=12600$, and $x \_3=2500$. In the ninth iteration also obtained s_8 $=5250,379873$ which is the excess material (residual material), $\mathrm{s} \_2=0.334295178, \mathrm{~s} \_3=$ $0,080230843, s_{-} 4=0,040115421, s_{-} 7=0,026743614$ and s_9 $=0,320930931$ which is use of material that exceeds the limit.

\section{CONCLUSION}

From the research result, it can be concluded that the total cost of production per month is the minimum production cost by producing square opak $14000 \mathrm{~kg}$, animal feed $12600 \mathrm{~kg}$ and opak bulk 2500 $\mathrm{kg}$, so in the month required production cost equal to $\mathrm{Rp} 93.320 .407,1$

\section{BIBLIOGRAPHY}

[1] Suprapto, J. 1983." Linear Programming" Edisi Kedua, Fakultas Ekonomi Universitas Indonesia. Jakarta.

[2] Handayani, Monica dan Dewi, Eka Kusuma, 2016. "Perencanaan Bahan Baku dan Hasil Produksi Menggunakan Metode Linier Programming Simpleks". Business Manajement Journal. Vol.12 (2), Hal 232-242.

[3] Rumahorbo, Rina Lusiana dan Mansyur, Abil. 2017. "Konsistensi Metode Simpleks Dalam Menentukan Nilai Optimum". Jurnal Karismatika. Vol. 3,(1), hal 36 - 46. 
[4] Chandra, Titin. 2015 "Penerapan Algoritma Simpleks dalam Aplikasi Penyelesaian Masalah Program Linier”. Jurnal TIMES. Vol. 4, (1), hal 18-21.

[5] Sukanta, Anwar Ilmar Ramadhan. 2016. "Simpleks Method Linear Program Application In Process Of Transition To Reduce Use Of Products In Polyster Material In Indonesia". International Journal Of Scientific \& Technology Research. Vol.5 (9), hal. 106-110.

[6] Dumairy. 20154Matematika Terapan Untu Bisnis Dan Ekonomi Edisi Kedua. Fakultas Ekonomika dan Bisnis UGM. Yogyakarta.

[7] Handayani, Monica dan Dewi, Eka Kusuma, 2016. "Perencanaan Bahan Baku dan Hasil Produksi Menggunakan Metode Linier Programming Simpleks”. Business Manajement Journal. Vol.12 (2), Hal 232-242.

[8] Kakiay, Thomas J. 2008. Pemrograman Linier Metode dan Problema. Andi. Yogyakarta.

[9] Lobo, J.Z. 2015 “Two Square Determinant Approach for Simplex Method”. Journal of Mathematics. Vol 11, (5). hal 01-04.

[10]Sarkoyo, Andi. 2016 "Metode Simpleks dalam Optimalisasi Hasil Produksi”. Informatics for Educators and Professionals. Vol. 1 (1), hal 27-36.

[11] Sukanta, Anwar Ilmar Ramadhan. 2016. "Simpleks Method Linear Program Application In Process Of Transition To Reduce Use Of Products In Polyster Material In Indonesia". International Journal Of Scientific \& Technology Research. Vol.5 (9), hal. 106-110.

[12] Wirdasari, Dian. 2009 "Metode Simpleks dalam Program Linier”. Jurnal Saintikom, Vol 6 (1), hal 276-285. 